\title{
Evaluación in vitro del efecto tóxico de una formulación comercial de glifosato de amonio sobre cinco especies representantes de diferentes hábitats y niveles tróficos
}

\section{In vitro assessment of toxic effects of a commercial formulation ammonium salt of glyphosate over five species representing different trophic levels and habitats}

Spósito, Martín (1), Espínola Moltedo, Julio César (1)

(1) Laboratorio de Bioensayos del Servicio de Evaluación de la Calidad y Control Ambiental, SECCA, Intendencia de Montevideo, IM

Contacto:marspo@gmail.com

RECIBIDO: 8/8/2016 - APROBADO: 15/10/2016

\begin{abstract}
Resumen
Se evaluó el efecto tóxico agudo y crónico in vitro de glifosato de amonio sobre especies que ocupan diferentes niveles tróficos y ambientes físicos: bacterioplanctónicos (Vibrio fischeri), fitoplanctónicos (Pseudokirchneriella subcapitata), zooplanctónicos (Daphnia magna), zoobentónicos (Hydra attenuata) y terrestre (Lactuca sativa). Se partió del preparado comercial herbicida TryGlif, a base de glifosato sal de amonio con una concentración de 430gr/L en peso de glifosato, más los llamados coadyuvantes (dispersantes, emulsionantes, solubilizantes, etc.) que poseen un potencial tóxico independiente y generalmente sinérgico con el principio activo. De las cinco especies analizadas, la de mayor sensibilidad al compuesto fue H. attenuata, con un valor de Concentración Letal 50\% (CL50) de 5,79 mg/L. De los cinco bioensayos considerados, la bacteria luminiscente $\mathrm{V}$. fischeri fue la que obtuvo una concentración de efecto $50 \%$ (CE50) mayor, alcanzando $37,28 \mathrm{mg} / \mathrm{L}$. El alga unicelular P. subcapitata tuvo una CL50 de 24,58 mg/L. La D. magna y la L. sativa presentaron valores similares de CL50 de 9,59 mg/L y 9,79 mg/L, respectivamente. Los efectos cuantificados sobre las diferentes especies analizadas aportaron un valor de referencia para siguientes investigaciones y se compararon con otros hallados en experimentos similares en la bibliografía internacional.

Palabras clave: TryGlif, herbicida, bioensayos, ecotoxicidad, aguas superficiales.
\end{abstract}

\begin{abstract}
We evaluated the acute and chronic toxic effect in vitro of a commercial brand of ammonium salt of glyphosate over five species that occupy different trophic levels and physical environments: bacterioplankton (Vibrio fischeri), phytoplankton (Pseudokirchneriella subcapitata), zooplankton (Daphnia magna), zoobenthos (Hydra attenuata) and terrestrial (Lactuca sativa). This investigation assesed the commercial preparation of herbicide Try Glif, based on ammonium salt of glyphosate with a concentration of $430 \mathrm{gr} / \mathrm{L}$ by weight of glyphosate, and inert compounds (dispersants, emulsifiers, solubilizers, etc.) with a potential toxicity is generally synergistic with the active principle. Among the five species tested the most sensitive to the compound was H. attenuata, with an LC50 of 5,79 mg/L. The luminescent bacterium V. fischeri was from the five bioassays that a median effective concentration scored higher, reaching $37,28 \mathrm{mg} / \mathrm{L}$. The unicellular alga P. subcapitata had an LC50 of $24,58 \mathrm{mg} / \mathrm{L}$. The D. magna and L. sativa had similar values of LC50 of $9,59 \mathrm{mg} / \mathrm{L}$ and $9,79 \mathrm{mg} / \mathrm{L}$ respectively. Quantified effects on different species analyzed provide a baseline for further investigations and were compared to those found in similar experiments in the international literature.

Keywords: TryGlif, herbicide, bioassays, ecotoxicity, superficial waters.
\end{abstract}

\section{Introducción}

El Glifosato (N-fosfonometil glicina) es un herbicida no selectivo y post emergente. Es uno de los herbicidas de mayor uso en la agricultura, por su probada eficacia, fácil manejo y su utilización en los modernos sistemas de cultivos (USDA, 2013). Su acción a nivel metabólico reside en la inhibición de la enzima 5-enolpiruvil chiquimato-3-fosfato sintetasa (EPSPS), 
que cataliza la reacción entre el ácido fosfoenolpirúvico y ácido 5-fosfochiquimico para sintetizar un precursor del ácido corísmico, en la vía del ácido chiquímico, que solo tiene lugar en plantas, bacterias y hongos (Martino, 1995). La enzima sobre la que actúa el glifosato es la responsable de la formación de los aminoácidos fenilalanina, triptofano y tirosina, que a la vez son utilizados para la formación de otros metabolitos secundarios.

La utilización de herbicidas basados en el glifosato ha aumentado considerablemente en cantidad, transformándose en nuestro país en el principal compuesto de su clase (REDES, 2012). Particularmente, su uso aumentó en el cultivo de algunas variedades a las cuales se les ha inducido genéticamente su resistencia (maíz, soja, entre otros). En Uruguay, un considerable aumento de la superficie cultivada y expuesta al uso de herbicidas ha tenido lugar en los últimos años. La superficie de cultivos de soja, el principal demandante de herbicidas a base de glifosato, aumentó desde el año 2003 al 2010 de 78,9 mil a 863,2 mil hectáreas, con un crecimiento de la producción que en ese mismo periodo pasó de 183 a 1816,8 mil toneladas anuales. Entre los años 2000 y 2011 las importaciones de herbicidas con base a este principio activo se multiplicaron por 7,5. Al final de este período la cantidad ascendía a 11253 toneladas anuales.

La investigación acerca de los efectos en las especies objetivo y no objetivo de herbicidas y pesticidas ha cobrado relevancia mundial, principalmente en los ejes de medicina, biodiversidad, evaluación de impacto ambiental y conservación de las aguas superficiales. Numerosos estudios (Alberdi, et al., 1996; Martin, et al., 2003; Martin y Ronco, 2006; Bonnet, et al., 2007; Sobrero, et al., 2007; Lallana, et al., 2013) han relevado la ecotoxicidad del principio activo glifosato, así como también de sus formulaciones comerciales, las cuales además del principio activo conllevan otros químicos mejoradores de la eficacia del glifosato (los más utilizados son los surfactantes, que colaboran en la adhesión del principio activo a los tejidos vegetales). En el año 1993 la US EPA clasificó al glifosato como una sustancia "no tóxica a moderadamente tóxica para aves, mamíferos e invertebrados acuáticos" (US EPA, 1993). Posteriores investigaciones han vinculado la exposición al glifosato o sus formulaciones con la incidencia de algunos tipos de cáncer en humanos (DeRoos, et al., 2003). Recientemente, la Agencia Internacional de Investigaciones sobre el Cáncer ha incorporado al glifosato en el grupo $2 \mathrm{~A}$ de sustancias probablemente carcinogénicas en humanos (Guyton, et al., 2015). Más allá de estas preocupaciones mayores que involucran a la salud humana, los posibles efectos del glifosato y sus formulaciones en el ambiente cobran una relevancia mundial y creciente.

Asimismo, se han desarrollado herramientas bioanalíticas (bioensayos) capaces de evaluar el efecto tóxico de los millones de productos químicos registrados en el Chemical Abstract Service (CAS), formulaciones comerciales complejas o muestras ambientales de las cuales se presume toxicidad. Estos ensayos biológicos realizados en el laboratorio utilizan especies que representan diferentes niveles tróficos y hábitats de los ecosistemas. Los resultados obtenidos en el laboratorio sobre muestras ambientales y productos químicos son extrapolados al ecosistema con la finalidad de predecir el deterioro del mismo (Eagleson, et al., 1990). Para esta investigación se utilizaron especies que representan diferentes niveles tróficos y ambientes acuáticos y terrestres (Tabla $\mathbf{1}$ ).

Basados en una de las formulaciones aún no comercializadas en nuestro país (Try Glif - $\mathrm{n}^{\circ}$ registro mgAP 4126) y en relevamientos previos de la presencia de glifosato en los cursos

\begin{tabular}{|c|c|c|}
\hline $\begin{array}{l}\text { Bioensayo/ } \\
\text { organismo }\end{array}$ & $\begin{array}{c}\text { Clasificación } \\
\text { por hábitat }\end{array}$ & $\begin{array}{l}\text { Nivel } \\
\text { trófico }\end{array}$ \\
\hline Daphnia magna & Zooplancton & $\begin{array}{c}\text { Heterótrofo/ } \\
\text { Consumidor } \\
\text { primario }\end{array}$ \\
\hline $\begin{array}{c}\text { Lactuca sativa } \\
\text { (var. Butercrunch) }\end{array}$ & Terrestre & $\begin{array}{l}\text { Autótrofo / } \\
\text { Productor } \\
\text { Primario }\end{array}$ \\
\hline $\begin{array}{l}\text { Pseudokirchneriella } \\
\text { subcapitata }\end{array}$ & Fitoplancton & $\begin{array}{c}\text { Autótrofo / } \\
\text { Productor } \\
\text { Primario }\end{array}$ \\
\hline Hydra attenuata & Zoobentos & $\begin{array}{c}\text { Heterótrofo/ } \\
\text { Consumidor } \\
\text { secundario }\end{array}$ \\
\hline Vibrio fischeri & Bacterioplancton & $\begin{array}{c}\text { Heterótrofo/ } \\
\text { Detritívoro }\end{array}$ \\
\hline
\end{tabular}

Tabla 1. Especies utilizadas, sus ambientes físicos y niveles tróficos.

de agua (Nuñez, et al., 2012; Peruzzo, et al., 2003; Aparicio, et al., 2013), el objetivo de este trabajo es determinar la toxicidad aguda y crónica de la formulación comercial TryGlif de glifosato en sal de amonio para cuatro especies de organismos acuáticos y la planta vascular terrestre Lactuca sativa (variedad Buttercrunch). La incorporación de esta especie sirve como referencia de las plantas vasculares, las cuales son uno de los objetivos directos del herbicida. La letalidad es una consecuencia extrema de la exposición de un organismo a un compuesto o una muestra dada, mientras que los organismos pueden verse afectados en una cantidad innumerable de variables biológicas. Desde el punto de vista de los ensayos, en algunos casos es posible medir la mortalidad y sobre ella se elaboran el índice de concentraciones letales (CL). En los casos donde no se puede comprobar la mortalidad directamente o que ésta no tenga lugar en el punto final al que arriban los ensayos, puede medirse la variación respecto al control de alguna variable biológica y estimar el efecto que sobre ella tiene la exposición a la muestra. De esta última estimación se obtienen valores de concentraciones de efecto o efectivas (CE).

El objetivo del trabajo es determinar la concentraciones letales (CL50) o de efecto 50\% (CE50) para cada una de las especies y comparar dicho valor con los valores conocidos de escorrentía en suelos donde fue utilizado el glifosato.

\section{Materiales y Métodos}

\section{Preparación de la solución problema}

La preparación de la solución del producto comercial de glifosato de amonio utilizado en los ensayos siguió los siguientes lineamientos:

- Las soluciones del producto analizadas fueron preparadas al momento de la realización de los diferentes bioensayos. Esta precaución se tomó para evitar el proceso natural de degradación vía hidrolítica que comienza a sufrir el principio activo en contacto con el agua. 
- Los solventes utilizados para la preparación de los diferentes tratamientos se ajustaron a las exigencias de los protocolos aplicados con modificaciones especiales en cuanto a reducir la dureza de los solventes (agua ultrapura) para evitar la precipitación del principio activo, precaución que surgió de las indicaciones sugeridas por el fabricante.

- El criterio para la realización de las diferentes series de concentraciones ensayadas a las que fueron expuestas las especies utilizadas se basó en el trabajo de Nuñez et al. (2012) que indica la concentración del principio activo hallada sobre un curso de agua adyacente a varias parcelas con y sin tapiz vegetal, donde se aplicó glifosato a las dosis usuales recomendadas y sobre las cuales, a su vez, se aplicó riego con la finalidad de simular lluvia. De dicho experimento se obtuvieron valores de runoff de entre 0,15 y $3,5 \mathrm{mg} / \mathrm{L}$ a 24 horas post-aplicación.

\section{Bioensayo con Lactuca sativa}

El ensayo con semillas de lechuga Lactuca sativa consiste en la exposición de la semilla a la solución problema midiendo la inhibición en el crecimiento radicular. El valor buscado es la concentración de efecto 50\% (CE50), que es la responsable de la disminución en un $50 \%$ del crecimiento de la raíz respecto a su control negativo con agua dura. El agua dura utilizada para el control negativo fue el agua de la marca comercial Nativa (Milotur S.A.): cloruros $12 \mathrm{mg} / \mathrm{L}$, calcio $64 \mathrm{mg} / \mathrm{L}$, magnesio $32 \mathrm{mg} / \mathrm{L}$, sodio $10,7 \mathrm{mg} / \mathrm{L}$ y potasio $1,1 \mathrm{mg} / \mathrm{L}$. En duplicado, 10 semillas de $L$. sativa fueron colocadas en placas de Petri con filtros de papel embebidos en $4 \mathrm{~mL}$ de la solución problema y puestas en oscuridad a $20 \pm 2{ }^{\circ} \mathrm{C}$ por 120 horas.

\section{Hydra attenuata}

Las Hydras fueron expuestas a distintas concentraciones de solución problema siguiendo los lineamientos del test agudo de Johnson et al. (1990) y Trottier, et al. (1997) por un plazo de 48 horas. Distribuidas de a tres hydras por poza en triplicado, en $4 \mathrm{~mL}$ de solución, al final del ensayo se cuenta la mortalidad de las hidras, entendida como la disgregación de los tejidos del animal o su inmovilidad luego de una acción mecánica.

\section{Daphnia magna}

Los ensayos con Daphnia magna se realizaron siguiendo los lineamientos de la UNE-ISO 6341:1996 (Asociación Española de Normalización y Certificación, 1996). Cinco ejemplares de D. magna fueron expuestos por duplicado en pozas de $10 \mathrm{~mL}$ de solución por 48 horas. Se identificó la mortalidad constatando la parálisis cardíaca de los individuos expuestos. Tanto para la $H$. attenuata como para la D. magna la incubación fue realizada a $20 \pm 2{ }^{\circ} \mathrm{C}$ con un fotoperíodo de 8 horas de luz, 16 de sombra y el control negativo fue el agua comercial Nativa. El valor hallado en ambos casos fue la concentración letal 50 (CL50), que es la concentración de solución problema capaz de matar al 50\% de los individuos expuestos.

\section{Pseudokirchneriella subcapitata}

El bioensayo con Pseudokirchneriella subcapitata es de los cinco bioensayos el único que evalúa la toxicidad a la exposición crónica. Consiste en la medición de la inhibición del crecimiento en un cultivo algal inoculado con distintas concentraciones de la solución problema. Las células fueron incubadas $(10 \mu \mathrm{L}$ cultivo algal a concentración conocida, $10 \mu \mathrm{L}$ de medio Bold y $200 \mu \mathrm{L}$ de muestra) en microplaca por un plazo de $72 \mathrm{hs} \mathrm{a}$ una temperatura de $24 \pm 2{ }^{\circ} \mathrm{C}$ y con luz permanente a 3000 lux por sextuplicado, con agitación permanente a $40 \mathrm{rpm}$. El control negativo se realizó inoculando el cultivo algal $(10 \mu \mathrm{L}$ cultivo algal y $10 \mu \mathrm{L}$ de medio Bold) en agua bicarbonatada ( $200 \mu \mathrm{L}$ de solución $\mathrm{NaHCO}_{3} 15 \mathrm{mg} / \mathrm{L}$ ). Terminado el ensayo, las células fueron contadas en hemocitómetro de Neubauer y al igual que en el caso de la $L$. sativa, la CE50 es aquella que reduce en un $50 \%$ el número de células vivas respecto al control negativo pasadas las $72 \mathrm{hs}$ del ensayo.

\section{$\operatorname{Microtox}^{\circledast}$}

El ensayo de Microtox corresponde a una técnica fotométrica que usa la respuesta a la exposición de tóxicos por parte de una bacteria marina bioluminiscente Vibrio fischeri. En la prueba la bacteria rehidratada es incubada durante $15 \mathrm{mi}$ nutos a $15{ }^{\circ} \mathrm{C}$ junto con la muestra y sus diluciones. Luego, la luminiscencia producida por la bacteria fue medida en un lector Microtox 500M. El ensayo realizado es el protocolizado como $81,9 \%$ Basic Test con nueve diluciones seriadas al 1/2, partiendo de una solución inicial de $434 \mathrm{mg} / \mathrm{L}$ de glifosato en sal de amonio del formulado TryGlif. La toxicidad de la muestra se expresa como la concentración en \% que inhibe el 50\% (CE50) de la producción de luz a los 15 minutos de exposición al contaminante (Dutka, 1989).

\section{Análisis estadísticos}

Para los ensayos de D. magna y $H$. attenuata el valor de la CL50 fue hallado por interpolación no lineal entre los valores de concentraciones próximos al 50\% en el programa TOXCALC (Environment Canada, 2007). Esto se debe a la falta de mortalidad parcial suficiente para realizar un ajuste por el método PROBIT.

La CE50 para el bioensayo con L. sativa se determinó utilizando el programa STATA 12.1 (www.stata.com). Para ello fue necesario el ajuste de una regresión no lineal paramétrica. El cálculo de la CE50 para P. subcapitata se realizó por el método de ICP "A linear interpolation method for sublethal toxicity" (Norberg-King, 1993). Para la determinación de la CE50 en el ensayo de Microtox fue utilizado el programa Microtox Omni Software, de acuerdo al protocolo de Basic Test. La concentración de muestra que produce una reducción del 50\% en la emisión de luz pasado los 15 minutos de exposición es la expresada como CE50.

\section{Resultados}

De las cinco especies analizadas la de mayor sensibilidad a la fórmula comercial de sal de amonio de Glifosato (Triglif ๑) fue la $H$. attenuata, con un valor de CL50 de 5,79 mg/L y un intervalo de confianza del 95\% (IC95\%) igual a: 3,39$6,78 \mathrm{mg} / \mathrm{L}$. La bacteria luminiscente $V$. fischeri fue de los cinco bioensayos el que obtuvo una mayor concentración de efecto 50, alcanzando 37,28 mg/L (IC95\%: 9,86 - 141,05 mg/L; $\mathrm{R}^{2}=0,82$ ). El alga unicelular $P$. subcapitata tuvo una CL50 de $24,58 \mathrm{mg} / \mathrm{L}$, con un intervalo de confianza de entre $21,61 \mathrm{y}$ $26,97 \mathrm{mg} / \mathrm{L}$. D. magna y $L$. sativa tuvieron valores similares de 
CL50 de 9,59 mg/L (IC95\%: 6,78 - 13,56 mg/L) y 9,79 mg/L (IC95\%: 8,17 - 11,41 mg/L; R²=0,89), respectivamente (Tabla 2). Es de destacar que el valor de la CL50 perteneciente a la D. magna ya se había alcanzado a las 24 hs del ensayo y se mantuvo permanente hasta las 48 hs después de finalizado.

\begin{tabular}{|c|c|c|}
\hline Especie & CL50/CE50 & IC95\% \\
\hline H. attenuata & 5,79 & $3,39-6,78$ \\
D. magna & 9,59 & $6,78-13,56$ \\
P. subcapitata & 24,58 & $21,61-26,97$ \\
L. sativa & 9,79 & $8,17-11,41$ \\
V. fischeri & 37,28 & $9,86-141,05$ \\
\hline
\end{tabular}

Tabla 2. Resultados obtenidos de la CL50/CE50 para las especies estudiadas en $\mathrm{mg} / \mathrm{L}$. El IC95\% es el intervalo de confianza del 95\%. ${ }^{*}$ Los valores de la CL50 en la D. magna son aquellos obtenidos a las 24 hs de iniciado el bioensayo.

\section{Discusión}

Algunos de los valores de las CL50/CE50 para las distintas especies se encontraron en el mismo orden que otros estudios con similares características (Tabla 3).

De los valores obtenidos de CL50 podemos afirmar que las especies H. attenuata, L. sativa y D.magna resultaron las más sensibles a la exposición al glifosato en sal de amonio TryGlif. Tanto el alga $P$. subcapitata como la bacteria fluorescente $V$. fischeri tienen una sensibilidad de efecto para este compuesto prácticamente de un orden menor respecto a las tres especies mencionadas anteriormente.

La alta disparidad de los valores de las CL50/CE50 para las diversas especies según los diferentes autores puede ser producto de las distintas formulaciones comerciales con las que se realizaron los bioensayos. A esta conclusión han llegado otros autores como Lallana et al. (2013), que encontraron diferencias significativas en el crecimiento radicular de $L$. sativa en el tratamiento con dos formulaciones comerciales distintas con la misma cantidad de principio activo.

Las formulaciones comerciales tienen además del principio activo glifosato una sal asociada y coadyuvantes (los más usados son los surfactantes). En cuanto a estos últimos se ha demostrado que pueden ser de mayor toxicidad que el principio activo, su sal asociada y sus principales productos de hidrolización y degradación en el medio acuoso (Martin, et al., 2003). Este aspecto debería redireccionar el área de investigación vinculada al uso del glifosato. Parece más importante evaluar el efecto tóxico y las consecuencias sobre el ambiente y la salud humana de los coadyuvantes que contienen las formulaciones de herbicidas basados en glifosato antes que el propio principio activo.

Los valores de la CL50/CE50 para las distintas especies están todas al menos un orden de magnitud por encima de las concentraciones encontradas del compuesto activo en aguas superficiales de escorrentía o de cursos de agua cercanos o linderos con los cultivos en los cuales se han aplicado herbicidas basados en el glifosato (Peruzzo, et al., 2008; Nuñez, et al., 2012; Aparicio, et al., 2013). De todas formas, esto no significa que las cantidades encontradas tanto de este tipo de herbicidas como de otros compuestos no tengan efectos en la composición y diversidad biológica de los ecosistemas acuáticos. Pequeñas variaciones en las poblaciones de una especie o de un nivel trófico pueden engendrar reacciones a distintos niveles que alteran drásticamente el funcionamiento global del sistema (Relyea, 2005; Pérez, et al., 2011). Del mismo modo, los efectos subletales, que implican una importante variación de algunas de las medidas vitales de los individuos (movilidad, reproducción, enraizamiento, entre otras), podrían engendrar una alteración de consecuencias sistémicas (Mensah, et al., 2012 y 2014). Este estudio evalúa mayoritariamente los efectos agudos que son consecuencia a una alta exposición a los tóxicos evaluados, pero no se puede predecir en base a estos resultados las consecuencias de una exposición subcrónica o crónica, la que suele tener una sensibilidad mayor (van Gestel, et al., 2001).

Es posible afirmar finalmente que estos ensayos se muestran como una herramienta biológica válida para evaluar los efectos tóxicos ambientales residuales producidos por esta formulación comercial a las concentraciones testeadas en este trabajo.

\begin{tabular}{|c|c|c|c|c|c|}
\hline & P. subcapitata & D. magna & H. atenuatta & L. sativa & V. fischeri \\
\hline Spósito et al. (2015) (mg/L de Gly en sal de amonio) & 24,58 & 9,59 & 5,79 & 9,79 & 37,28 \\
\hline IUPAC (mg/L de Gly) & 4,4 & 40 & & & \\
\hline Suprenant (1981) (mg/L de Gly en IPA sal) & & 5,3 & & & \\
\hline Alberdi (1996) (48\%de Gly en sal de IPA y 15\% de surfactante) & & 95,96 & & & \\
\hline Martin y Ronco (2006) (RoundUp Max mg/L de p.a.) & & & & 7,3 & \\
\hline Martin (2003) (mg/L de Gly en sal de amonio) (RoundUp Max mg/L de p.a.) & 41 & & & & 162 \\
\hline Lallana (2013) (mg/L de Gly en sal de amonio) & & & & 668,2 & \\
\hline Folmar et al. (1979) (RoundUp Max mg/L de p.a.) & & 3 & & & \\
\hline
\end{tabular}

Tabla 3. Valores de CL50/ CE50 hallados en este trabajo y por otros autores para los respectivos compuestos que contienen glifosato como principio activo. GLY en IPA sal: glifosato en sal de isopropilamina. RoundUp y RoundUp Max son productos comerciales con el principio activo glifosato. 


\section{Reconocimientos}

Se agradece al Quím. Andrés Pérez por proporcionar el glifosato utilizado en el presente estudio y contribuir en la determinación del diseño experimental.

Se agradece al Ing. Agr. Saturnino Nuñez por proporcionar información de antecedentes de estudios de campo y contribuir en la determinación del diseño experimental.

\section{Referencias}

Asociación Española de Normalización y Certificación, 1996. UNE-ISO 6341: Calidad de agua. Determinación de la inhibición de la movilidad de Daphnia magna Straus (Cladocera, Crustacea). Ensayo de toxicidad aguda. Madrid: AENOR.

Alberdi, J.L., Sáenz, M.E., Di Mazio, W.D., Tortorelli, M.C., 1996. Comparative acute toxicity of two herbicides, paraquat and glyphosate, to Daphnia magna and D.spinulata. En: Bulletin of Environmental Contamination and Toxicology, 57, pp.229-235.

Aparicio, V.C., et al., 2013. Environmental fate of glyphosate and aminomethylphosphonic acid in surface waters and soil of agricultural basins. En: Chemosphere, 93(9), pp.1866-1873.

Bonnet, J.L., Bonnemoy, F., Dusser, M. y Bohatier, J., 2007. Assessment of the potential toxicity of herbicides and their degradation products to non target cells using two microorganisms, the bacteria Vibriof ischeri and the ciliate. En: Tetrahymena pyriformis, Environ. Toxicol., 22(1), pp.78-91.

De Roos, A. J., Zahm, S., Cantor, K., Weisenburger, D., Holmes, F., Burmeister, L. y Blair, A., 2003. Integrative assessment of multiple pesticides as risk factors for nonHodgkin's lymphoma among men. En: Occupational and Environmental Medicine, 60(9), e11.

Dutka, B.J., 1989. Methods for microbiological and toxicological analysis of waters, wastewaters, and sediments. Ontario: Ed. Rivers Research Branch, NWRI, CCIW, Burlington.

Eagleson, K.W., Lenat, D.L., Ausley, L.W. y Winborne, F.B., 1990. Comparison of measured instream biological responses with responses predicted using the Ceriodaphnia dubia chronic toxicity test. En: Environ. Toxicol. Chem., (9), pp.1019-1028.

Environment Canada, 2007. Guidance document in statistical methods for environmental toxicity tests. Ottawa: Environment Canada. (Report EPS 1/RM/46).

Folmar, L.C., Sanders, H.L. y Julin, A.M., 1979. Toxicity of the herbicide glyphosate and several of its formulations to fish and aquatic invertebrates. En: Arch. Environ. Contam. Toxicol, 8(3), pp.269-278.

Guyton, K.Z., Loomis, D., Grosse, Y., El Ghissassi, F., Benbrahim-Tallaa, L., Guha, N., Scoccianti, C., Mattock, H. y Straif, K., 2015. Carcinogenicity of tetrachlorvinphos, parathion, malathion, diazinon, and glyphosate. En: Lancet Oncol, 16(5), pp.490-1.

Johnson, E.M., Gabel, B.E.G, Newman, L.M. y Giacobbe, R., 1990. The Hydra assay manual. A practical guide to supplies, techniques and mechanics of the assay. Filadelfia: Department of Anatomy, Daniel Baugh Institute, Jefferson Medical College.
Lallana, María del C., et al., 2013. Determinación de reducción del crecimiento radical (CE50) por una formulación de glifosato utilizando lechuga y trigo como especies bioindicadoras [En línea]. En: Rev. Fac. Cienc. Agrar., Univ. Nac. Cuyo, 45(1). [Consulta: enero de 2015]. Disponible en: http://revista.fca.uncu.edu.ar/images/stories/ pdfs/2013-01/T45_01_13_Lallana.pdf

Martin, M.L., Sobrero, M.C., Rivas, C., Rimoldi, F. y Ronco, A., 2003. Impacto del uso de pesticidas asociado a la siembra directa sobre especies no-blanco: flora riparia y acuática. En: Memorias de la Conferencia Internacional Usos Múltiples del Agua. Cartagena de Indias: Universidad del Valle.

Martin, M.L. y Ronco, A.E., 2006. Effects of mixtures of pesticides used in the direct seedling technique on non-target plant seeds. En: Bulletin of Environmental Contamination and Toxicology, 77(2), pp.228-236.

Martino, D. L., 1995. El herbicida glifosato: su manejo más allá de la dosis por hectárea. Montevideo: INIA. (Serie Técnica, no. 61).

Mensah, P.K., Muller, W.J., y Palmer, C.G., 2011. Acute toxicity of Roundup (R) herbicide to three life stages of the freshwater shrimp Caridina nilotica (Decapoda: Atyidae). En: Phys Chem Earth, 36, pp.905-909.

Mensah, P.K., Palmer, G. y Muller, Wilhelmine J., 2014. Using growth measures in the freshwater shrimp Caridina nilotica as biomarkers of Roundup pollution of South African freshwater systems. En: Phys Chem Earth, (50-52), pp.262-268

Mensah, Paul, K., C., Palmer, G. y Wilhelmine J. Muller, 2014. Lethal and sublethal effects of pesticides on aquatic organisms: the case of a freshwater shrimp exposure to Roundup ${ }^{\oplus}$ [En línea]. Soloneski, Sonia ed. Pesticides toxic aspects. [s.l.]: InTech. ISBN: 978-953-51-1217-4. DOI: 10.5772/57166. [Consulta: noviembre de 2015]. Disponible en: http://www.intechopen.com/books/ pesticides-toxic-aspects/lethal-and-sublethal-effectsof-pesticides-on-aquatic-organisms-the-case-of-afreshwater-shrimp-expo

Norberg-King, T.J., 1993. A linear interpolation method for sublethal toxicity: the inhibition concentration (ICP) approach. Minnesota: US EPA. (Technical report 03-93).

Nuñez, S., Maeso, D., Franchi, S., Fiorentino G., Chouhy A., Ferrazzini, H., Fernandez A., Takahashi, S. y Gonda, G., 2012. Evaluación del escurrimiento superficial de plaguicidas en suelos agrícolas representativos del país. Montevideo: INIA, JICA, mgAP

Pérez, G.P., Vera, M.S. y Miranda, L., 2011. Effects of herbicide glyphosate and glyphosate-based formulations on aquatic ecosystems [En línea]. En: Kortekamp, A. ed., Herbicides and environment. [s.l.]: InTech. ISBN 978-953307-476-4. DOI: 10.5772/12877. [Consulta: noviembre de 2015]. Disponible en: http://www.intechopen.com/ books/herbicides-and-environment/effects-of-herbicideglyphosate-and-glyphosate-based-formulations-onaquatic-ecosystems

Peruzzo, P., Marino, D., Cremonte, C., da Silva, M., Porta, A. y Ronco, A., 2003. Impacto de pesticidas en aguas superficiales y sedimento asociado a cultivos por siembra directa. Memorias Conferencia Internacional Usos del Agua, Cartagena de Indias. Cartagena de Indias: IWA. pp.135-142.

Peruzzo, P.J., Porta, A.A., Ronco, A.E., 2008. Levels of glyphosate in surface waters, sediments and soils 
associated with direct sowing soybean cultivation in north pampasic region of Argentina. En: Environ. Pollut., 156(1), pp.61-66.

REDES, 2012. Crecimiento de la agricultura y el uso de agrotóxicos en Uruguay [En línea]. Montevideo: Redes Amigos de la Tierra - Uruguay. [Consulta: noviembre de 2015]. Disponible en:

http://www.redes.org.uy/wp-content/uploads/2014/01/FolletoAgr-y-Agrotoxicos-Redes-WEB.pdf

Relyea, R. A., 2005. The impact of insecticides and herbicides on the biodiversity and productivity of aquatic communities. En: Ecol. Appl., 15(2), pp.618-627.

Sobrero, M.C., Rimoldi, F., Ronco, A.E., 2007. Effects of the glyphosate active ingredient and a formulation on Lemma gibba L. at different exoposure levels and assessment end-points. En: Bull. Environ. Contam. Toxicol.,79, pp.537-543.

Trottier, S., Blaise C., T. Kusui y E.M. Johnson, 1997. Acute toxicity Assessment of Aqueous Samples Using a
Microplate-based Hydra attenuata Assay. En: Environmental Toxicology and Water Quality, 12, pp.265-271.

U.S. Department of Agriculture Economic Research Service, USDA ERS, 2013. Adoption of genetically engineered crops in the U.S. Washington, DC. [En línea]. Washington: USDA ERS. [Consulta: octubre de 2015]. Disponible en: www.ers.usda.gov/data-products/ adoption-of geneticallyengineered-crops-in-the-us.aspx\#. UeRCQW0fhIQ

US EPA, 1993. R.E.D. Facts: glyphosate. Washington: US EPA, p.7.

US EPA, 1996. Ecological effects test guidelines. Seed germination/root elongation toxicity test. Minnesota: US EPA. (OPPTS 850.4200).

van Gestel, C. A. M., van der Waarde, J. J., Derksen, J. G. M., van der Hoek, E. E., Veul, M. F. X. W., Bouwens, S., Rusch,B., Kronenburg, R., y Stokman, G. N. M., 2001. The use of acute and chronic bioassays to determine the ecological risk and bioremediation efficiency of oil-polluted soils. En: Environ. Toxicol. Chem, 20, pp.1438-1449. 